\title{
Proton radiotherapy dose perturbations caused by esophageal stents of varying material composition are negligible in an experimental model
}

Authors

Institutions

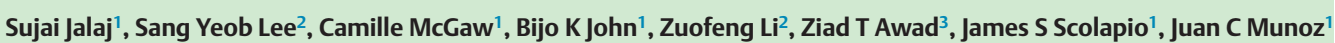

${ }^{1}$ Division of Gastroenterology, Department of Medicine, University of Florida, Jacksonville College of Medicine, Jacksonville, Florida, United States

${ }^{2}$ Department of Radiation Oncology, University of Florida, Proton Therapy Institute, Jacksonville, Florida, United States

${ }^{3}$ Department of Surgery, University of Florida, Jacksonville College of Medicine, Jacksonville, Florida, United States submitted 20. March 2014 accepted after revision 8. September 2014

\section{Bibliography}

Dol http://dx.doi.org/

10.1055/s-0034-1390709

Published online: 24.10.2014

Endoscopy International Open 2015; 03: E46-E50

(c) Georg Thieme Verlag KG

Stuttgart · New York

E-ISSN 2196-9736

\section{Corresponding author}

\section{Sujai Jalaj, MD}

Division of Gastroenterology,

Department of Medicine

University of Florida,

Jacksonville College of Medicine

655 West 8th Street, B0138-

C290

Jacksonville, FL 32209

Fax: +1-904-244-2165

sujai.jalaj@jax.ufl.edu
Background: Self-expanding metal and plastic esophageal stents (SEMS and SEPS, respectively) are used in conjunction with chemoradiation for palliation of malignant dysphagia. To date, the dosimetric effects of stents undergoing proton radiotherapy are not known.

Study aim: To investigate the proton radiotherapy dose perturbations caused by esophageal stents of varying designs and materials undergoing external beam treatment for esophageal cancer. Patients and methods: Simulated clinical protocol. Solid acrylic phantom was used to mimic the esophageal tissue environment. Stents made of nitinol, stainless steel and polyester were tested. Proton beam dose of $2 \mathrm{~Gy}-\mathrm{E}$ was delivered to each stent in a single anterior to posterior field. Film and image based evidence of dose perturbation were main outcomes measured.

\begin{tabular}{|c|c|}
\hline \multicolumn{2}{|c|}{ Abbreviations } \\
\hline ICRU & $\begin{array}{l}\text { International Commission on Radiation } \\
\text { Units and Measurements }\end{array}$ \\
\hline SEMS & self-expanding metal stent \\
\hline DPC & self-expanding plastic esophageal stent \\
\hline
\end{tabular}

\section{Introduction}

$\nabla$

Esophageal cancer is the eighth most common cancer worldwide; an estimated 484000 new esophageal cancer patients were diagnosed worldwide and approximately 407000 deaths worldwide occurred in 2008 [1]. The American Cancer Society estimates that in the United States approximately 17990 new patients of esophageal cancer have been diagnosed in 2013 and approximately 15210 deaths have occurred [12]. Most patients suffering from esophageal cancer presents with dysphagia due to luminally obstructive tumor. Endoluminal esophageal stenting
Results: Only the stainless steel and plastic stents demonstrated slight overall dose attenuations ( $-0.5 \%$ and $-0.4 \%$, respectively). All the nitinolbased stents demonstrated minimal overall dose perturbations ranging from $0.0 \%$ to $1.2 \%$. Negligible dose perturbations were observed on each of the stent surfaces proximal to the radiation source, ranging from $-0.8 \%$ (stainless steel stent) to $1.0 \%$ (nitinol stent). Negligible dose effects were also observed on the distal surfaces of each stent ranging from $-0.5 \%$ (plastic and stainless steel stents) to $1.0 \%$ (nitinol stent).

Conclusion: Proton radiotherapy dose perturbations caused by stents of varying designs and material composition are negligible. Negligible dose perturbation is in keeping with the inherent advantage of proton therapy over traditional radiotherapy composed of photons - given its relative large mass, protons have little side scatter.

with self-expanding metal or plastic stents (SEMS or SEPS) are often used for palliation of malignant dysphagia either alone or before definitive or palliative chemoradiotherapy. Studies of radiotherapy and endoluminal esophageal stent placement have revealed that SEMS or SEPS have a more immediate effect than radiotherapy. Radiotherapy can take weeks for symptomatic improvement, however, in contrast to esophageal stenting, the resolution of dysphagia is more lasting [3-6].

In conventional photon radiotherapy of esophageal cancers, the presence of esophageal stents is known to cause a dose perturbation at the interface between the stent and surrounding esophageal tissue. This dose perturbation is primarily due to secondary electrons and scatter caused by the metallic components within the stent. Previous studies using tissue-mimicking phantom models and Monte Carlo simulations have shown that these dose perturbations can include both an increase and decrease in radiation dose to the sur- 


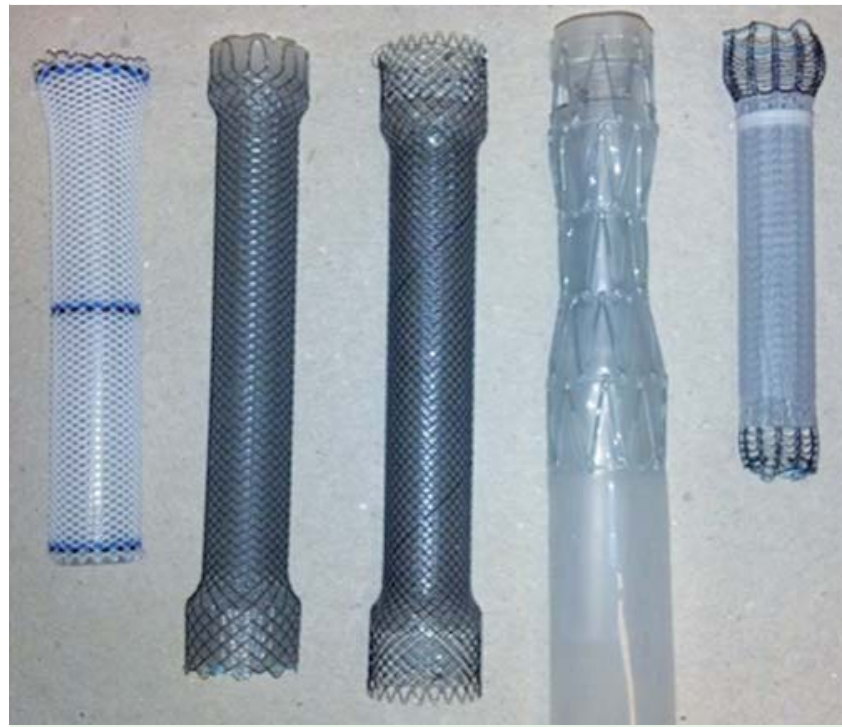

Fig. 1 Esophageal stents analyzed in this study (from left to right) included Polyflex, Wallflex, Evolution, Z-Stent, and Ultraflex.

rounding tissue, depending on the material composition of the esophageal stent [7-10]. However, these studies only examined one type of radiation therapy-photon therapy. To date, the dosimetric effects of stents undergoing proton radiotherapy are not known.

Proton therapy is a type of external beam radiotherapy using ionized radiation. Protons are positively charged particles. These charged particles damage the DNA of cells, thereby causing their death or interfering with their ability to proliferate. A number of treatment plan comparison studies have demonstrated that proton irradiation offers a far better localized treatment and smaller irradiated volumes of normal tissue when compared to conventional photon irradiation techniques [11 - 15]. Thus, the chief advantages of proton therapy are the ability to provide more localized radiation dosage to the tumor volume and to minimize radiation dose to surrounding healthy tissue when compared to photon therapy.

The aim of this study is to measure the dosimetric perturbations caused by a variety of different esophageal SEMS and SEPS in a tissue-mimicking phantom model undergoing proton therapy.

\section{Materials and methods}

$\nabla$

This study was exempt from Institutional Review Board review because patient information and animal or human subjects were not used. Five esophageal stents of various designs and materials were examined in the setting of proton radiotherapy. Commercially available esophageal stents with mesh composi-

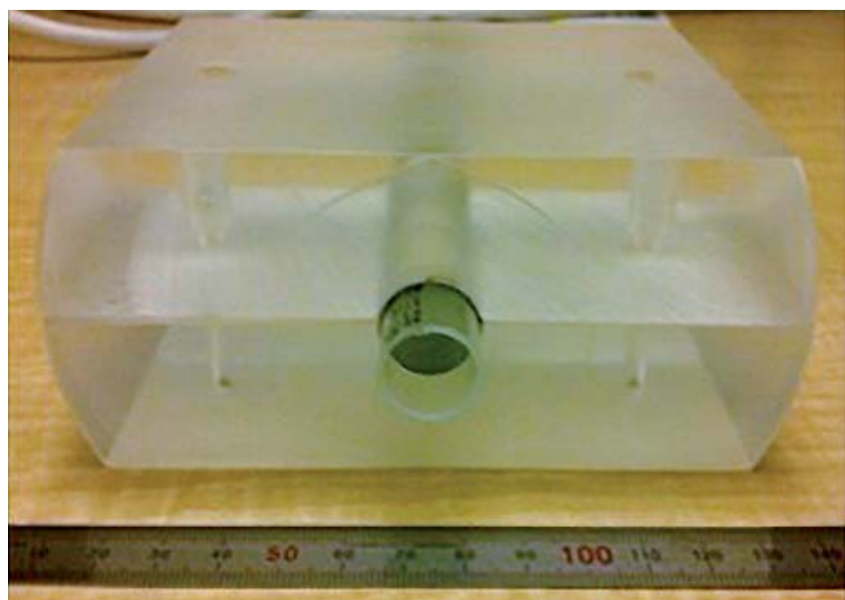

Fig. 2 Acrylic phantom.

tion made of nitinol (Evolution, Wallflex and Ultraflex), stainless steel (Cook Z-Stent), polyester (Polyflex) and covered with either polyurethane (Ultraflex), silicone (Evolution, Wallflex and Polyflex), or polyethylene (Cook Z-Stent) were tested ( $\mathbf{F i g .} 1$ and - Table 1). Of note, the Polyflex stent also contained some tungsten radiopaque markers positioned on three narrow radio-opaque bands, included for fluoroscopic localization purposes during typical endoscopic deployment. Each tungsten localization marker was approximately $2 \mathrm{~mm}$ in diameter, and each band contained $0.15-0.22 \mathrm{~g}$ of tungsten.

To mimic the tissue environment of the esophagus, we used an acrylic solid phantom (University of Florida Proton Therapy Institute, Jacksonville, Florida) measuring $6 \times 6 \times 6 \mathrm{~cm}$ with a $20 \mathrm{~mm}$ diameter bored cylindrical core ( $\bullet$ Fig. 2). A phantom is a water or plastic substitute for tissue in radiotherapy measurements. The solid material of our phantom had radiological characteristics similar to water and has commonly been used as a dosimetry model for radiotherapy beam calibrations as well measurements intended to simulate human esophageal tissue irradiation [16, 17]. Our phantom was sectioned in half along the central axis of the bore for ease of placement of the esophageal stents.

To qualitatively measure delivered radiation doses, special high resolution film, GafChromic EBT3 (Ashland, Covington, Kentucky), was used. Each piece of radiochromic film $(5.8 \times 10 \mathrm{~cm})$ was wrapped around the outer circumference of each stent. An air-filled $12 \mathrm{cc}$ plastic syringe was then placed within the lumen of a stent. The film, stent and syringe were then placed into the $18 \mathrm{~mm}$ diameter cylindrical core, sandwiched between two 3$\mathrm{cm}$ layers of our acrylic phantom ( $\bullet$ Fig. 3 ), and exposed to a perpendicular proton beam from a proton cyclotron (IBA C230, Belgium; $\bullet$ Fig. 4). An empty phantom (film wrapped around air-filled syringe without a stent) was used as a control setup for each stent.

Table 1 Stent Characteristics.

\begin{tabular}{|lllll|}
\hline Stent Name & Manufacturer & Stent Base Material & Stent Cover Material & Design \\
\hline Evolution & Cook Medical & Nitinol & Silicone & Partially covered, nitinol braided mesh \\
\hline Polyflex & Boston Scientific & Polyester & Silicone & Fully covered, polyester mesh \\
\hline Ultraflex & Boston Scientific & Nitinol & Polyurethane & Partially covered, nitinol weave \\
\hline Wallflex & Boston Scientific & Nitinol & Silicone & Fully covered, nitinol braided mesh \\
\hline Z-Stent & Cook Medical & Stainless Steel & Polyethylene & Fully covered, stainless steel mesh \\
\hline
\end{tabular}




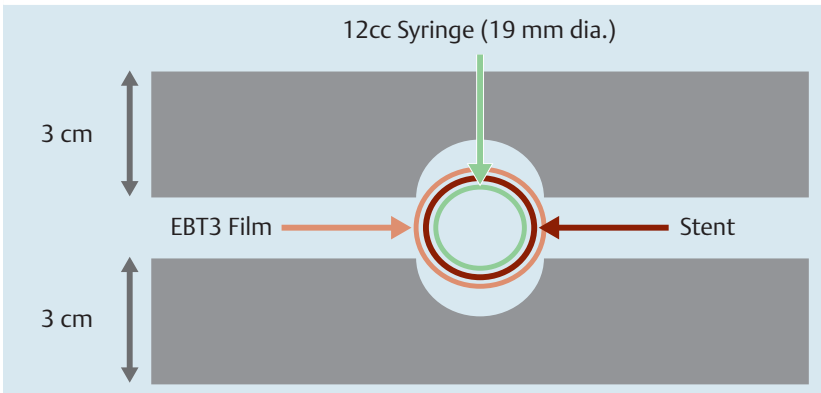

Fig. 3 Illustration of acrylic phantom setup. An air-filled 12 cc syringe was placed within the lumen of each stent and radiochromic film (GafChromic EBT3; Ashland, Covington, Kentucky) was wrapped around the outer circumference of each stent. The film, stent, and syringe were then placed into the $20 \mathrm{~mm}$ diameter cylindrical core, sandwiched between two 3-cm layers of our acrylic phantom.

A proton dose of $2 \mathrm{~Gy}-\mathrm{E}$ was delivered to the phantom with the film-stent-syringe sandwich in a single anterior-posterior field. This arrangement provided for measurement of both forward and back scatter in the same model. A 2 Gy-E dose was chosen to use the expected linear range of the radiochromic film, allowing for all dose perturbations to be accurately measured.

All measurements were performed twice to verify the reproducibility of the results. To measure delivered radiation doses, exposed radiochromic film was read with a film scanner (Epson Expression 10000XL: Red channel). The results were analyzed with dosimetry software (FilmQA Pro 3.0; Ashland, Kentucky). An experienced radiation physicist performed all measurements.

All films were processed to quantify the dose perturbations next to the stents at locations proximal and distal to the radiation source. The piece of radiochromic film proximal to the radiation source was used to measure the back scattered radiation, and the piece of film distal to the radiation source was used to measure the radiation dose perturbations caused by the absorption and scatter properties of the stent.

\section{Results}

Each of the five stents demonstrated some degree of minimal dose perturbation. As mentioned earlier, when the radiochromic film was wrapped around the stent, one portion of the stent was closer to the radiation source (proximal side) and experienced back scattered radiation enhancement. The other portion of the film was on the opposite region (distal side), which was expected to show slightly lower radiation dose because it was shadowed by the proximal surface of the stent and because it was slightly farther from the radiation source. Consequently, each half of the film was designated as proximal and distal relative to the radiation source.

- Fig. 5 provides a qualitative and visual sense of the minimal degree of proton dose perturbation captured by GafChromic films. The control film (phantom with radiochromic film wrapped around air-filled syringe without stent) exhibited uniform dose distributions at locations proximal and distal to the radiation source when irradiated with a proton dose of $2 \mathrm{~Gy}-\mathrm{E}$. The percent difference between the maximum and minimum dose values for the control film was $1.3 \%$. We assumed that this dose spread was inherent to the radiochromic film, and that it was indicative of the dose spread that would also be observed in the exposed stent films.

The three nitinol stents (Ultraflex, Evolution, and Wallflex) demonstrated no overall dose perturbation to minimal overall dose enhancement ranging from $0.0 \%$ to $1.2 \%$ ( $\bullet$ Fig. 5).

Of the five esophageal stents, only the Z-Stent and Polyflex demonstrated average dose attenuations and distinct, focal areas of "cold spots" on radiochromic film analysis. The dose attenuation observed in the Z-Stent occured in repeating patterns ( $\bullet$ Fig.5).
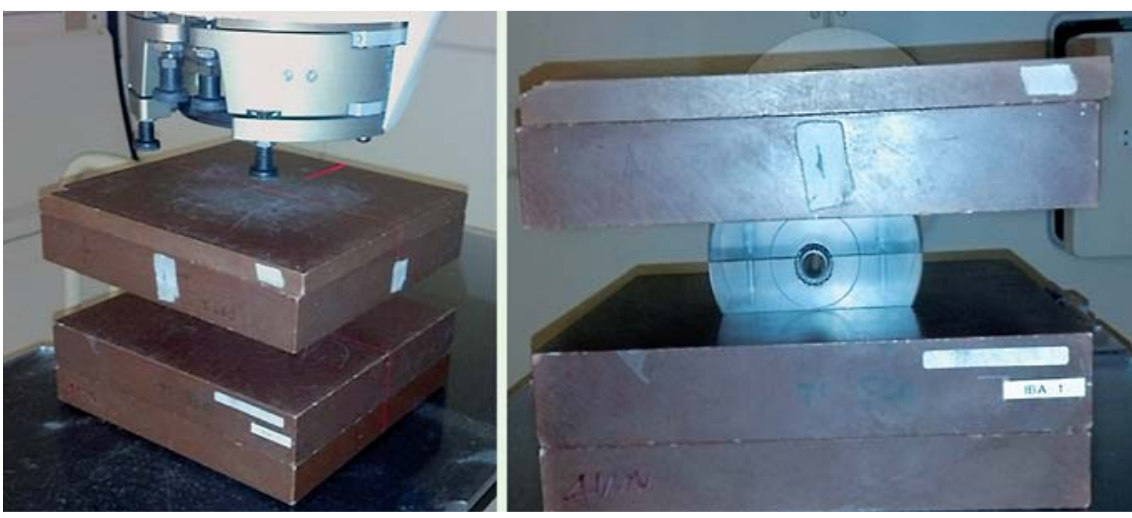

Fig. 4 Two views of a proton cyclotron.
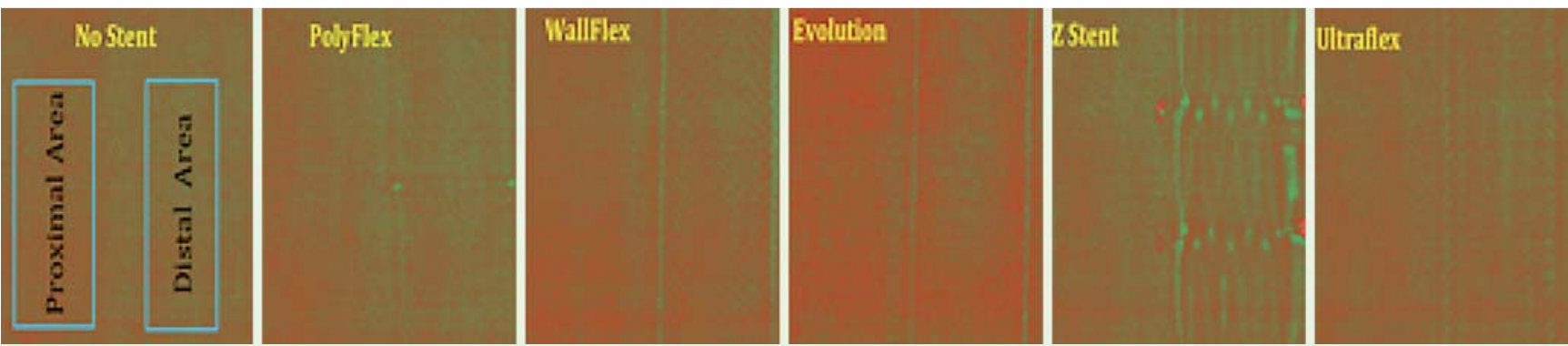

Fig.5 Patterns of dose perturbations measured with radiochromic film at locations proximal and distal to the radiation source for each of the five stents. Decreased pixel intensity (lighter color shade) corresponds to decreased dose. 


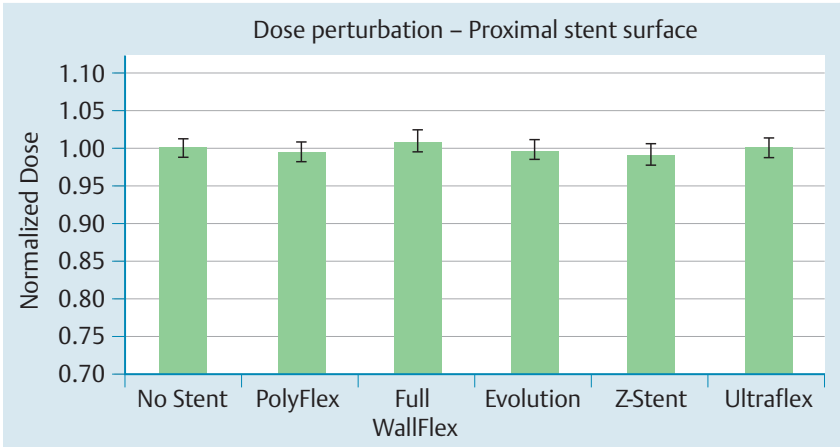

Fig. 6 Proximal stent surface dose perturbation. All five stents demonstrated roughly the same minimal degree of dose perturbation on the proximal stent surface (relative to radiation source).

The nonmetallic Polyflex stent demonstrated distinct, focal areas of cold spots or dose attenuation that was readily observed on radiochromic film ( $\bullet$ Fig.5). The average percent decrease in proton dose in these focal cold spot regions was $11.6 \%$ and $14.5 \%$ for the Polyflex and Z-Stent, respectively.

Dose perturbations measured for each stent type at locations proximal and distal to the radiation field are shown in $\bullet$ Fig. 6 and $\odot$ Fig. 7, respectively. The dose perturbations on the proximal and distal surfaces of each stent were minimal. Furthermore, when each stent was compared to the other, it was evident that all five stents demonstrated roughly the same minimal degree of dose perturbation on its respective proximal and distal surfaces $(\bullet$ Table2). Negligible dose perturbations were observed on each of the stent surfaces proximal to the radiation source, ranging from $-0.8 \%$ (Z-Stent) to $1.0 \%$ (Wallflex). Negligible dose effects were observed on the distal surfaces of each stent, ranging from $-0.5 \%$ (Polyflex and Z-Stent) to $1.0 \%$ (Wallflex). Consequently, the average dose perturbation for each individual stent was minimal ( $\bullet$ Fig.8).

\section{Discussion}

Radiation treatment of esophageal cancer causes much morbidity because of the proximity of organs such as the heart and lung. Although the dosimetric advantage of protons is clear, the reported clinical experience using proton radiotherapy in the treatment of esophageal cancers is limited. A recent study conducted by Welsh et al has shown that when compared to intensity modulated photon radiation therapy, intensity modulated proton therapy for distal esophageal cancer leads to considerable reductions in the dose to neighboring thoracic organs such as heart, lungs, and liver [18]. Another study by Lin et al also demonstrates that proton radiotherapy for esophageal cancer is associated with few acute treatment-related toxicities and perioperative morbidities [19].

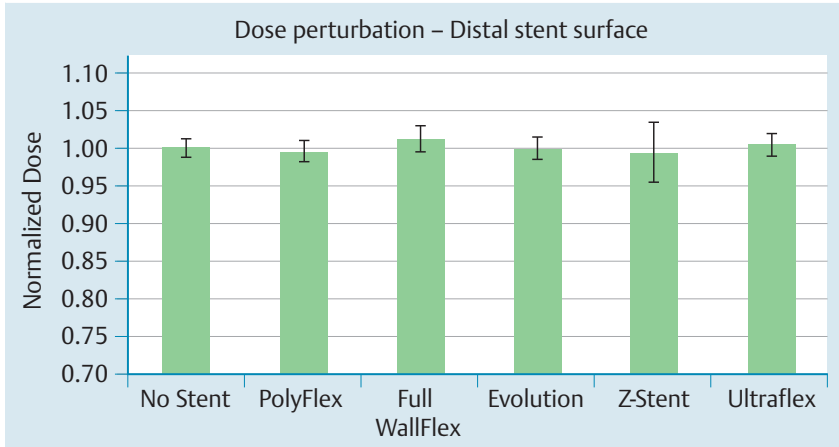

Fig. 7 Distal stent surface dose perturbation. All five stents demonstrated roughly the same negligible degree of dose perturbation on the distal stent surface (relative to radiation source).

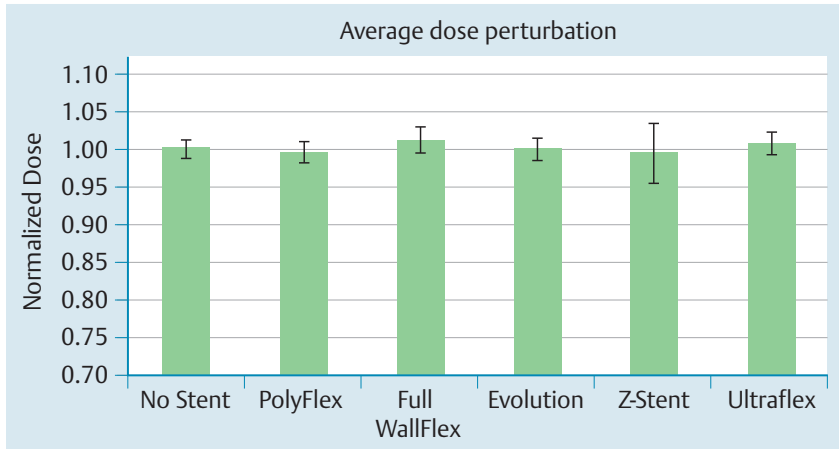

Fig. 8 Average dose perturbation for each individual stent is negligible. The uncertainty data for each measurement are based on the maximum percent difference observed from the corresponding control film.

Because proton therapy appears to be a newer radiation technique to limit radiation toxicity, it is important to understand the role of proton radiotherapy in the setting of esophageal stents. To date, the dosimetric effects of esophageal stents undergoing proton radiotherapy are not known. Prior studies conducted by Chen et al, Atwood et al, and Abu Dayyeh et al have demonstrated that radiation dose perturbations do occur in esophageal stents of various materials and designs when undergoing traditional radiation therapy using photons $[8-10]$.

For treatment with a single external proton beam, a negligible average dose perturbation ranging from $-0.5 \%$ to $1.2 \%$ is observed among esophageal stents of varying designs and material composition. This small magnitude of dose perturbation is in keeping with the inherent advantage of proton therapy - given their relatively large mass, protons have little side scatter. The minimization of side or back scatter is the major reason there is minimal to no dose enhancement observed at the proximal surface of the stents. This is in stark contrast to the studies of Chen et al and Atwood et al that reported dose enhancements at the

Table 2 Dose perturbation ranges measured from the five esophageal stents at locations proximal and distal to the proton radiotherapy source.

\begin{tabular}{lccccc} 
& Polyflex & Wallflex & Evolution & Z-Stent & Ultraflex \\
\hline Proximal stent surface & $-4.8 \% \pm 1.3 \%$ to & $-4.2 \% \pm 1.3 \%$ to & $-4.5 \% \pm 1.3 \%$ to & $-5.3 \% \pm 1.3 \%$ to & $-4.1 \% \pm 1.3 \%$ to \\
& $5.4 \% \pm 1.3 \%$ & $6.2 \% \pm 1.3 \%$ & $4.7 \% \pm 1.3 \%$ & $5.2 \% \pm 1.3 \%$ & $5.3 \% \pm 1.3 \%$ \\
\hline Distal stent surface & $-11.2 \% \pm 1.3 \%$ to & $-8.8 \% \pm 1.3 \%$ to & $-6.3 \% \pm 1.3 \%$ to & $-14.1 \% \pm 1.3 \%$ to & $-5.8 \% \pm 1.3 \%$ to \\
& $5.8 \% \pm 1.3 \%$ & $9.1 \%$ to $1.3 \%$ & $6.0 \% \pm 1.3 \%$ & $9.7 \% \pm 1.3 \%$ & $6.1 \% \pm 1.3 \%$
\end{tabular}

The uncertainty data for each measurement are based on the maximum percent difference observed from the corresponding control film. 
proximal surfaces of all stents undergoing treatment with traditional photon radiotherapy $[8,9]$.

In contrast, the Polyflex and Z-Stent demonstrated an overall average dose attenuation of $-0.4 \%$ and $-0.5 \%$, dose attenuation at its distal stent surfaces, and distinct cold spots. The dose attenuation observed in the Z-Stent occurs in repeating patterns due to its steel composition and mesh design. Focal cold spots within the Z-Stent were observed primarily in areas in which metallic mesh overlap was readily observed on the radiochromic film ( $\bullet$ Fig. 5). The nonmetallic Polyflex stent does not illustrate a pattern of dose perturbation throughout the stent. However, this stent contains three radiopaque tungsten markers that circumscribe the stent at the top, middle, and bottom. These radiopaque tungsten markers produce focal areas of cold spots or dose attenuation that were readily observed on the radiochromic film (৫ Fig.5).

The dose attenuation and focal areas of cold spots observed on the Polyflex and Z-Stent are due to the presence of high atomic number (Z) materials in each stent (eg, stainless steel in Z-Stent and tungsten markers in Polyflex). High $Z$ materials are known to scatter protons through larger angles than low $\mathrm{Z}$ materials. Although higher $\mathrm{Z}$ materials are more likely to scatter proton beams, they are less likely to decrease proton energy, which is why the overall average proton dose was similar in the Z-Stent and Polyflex when compared to the three nitinol-based stents. Although this study only analyzed the dosimetric impact of esophageal stents in a single radiation field, we hypothesize that the instances of hot and cold spots would increase with the addition of multiple fields from different gantry angles, which are often used in intensity modulated proton therapy. When two opposed beams are used, and the stent surfaces are at the same depth with regard to each beam, there is a small possibility that the hot spot created by one beam would be cancelled by a cold spot generated by the opposing beam. However, this situation is not likely because the use of opposed beams in intensity modulated proton therapy is not common in clinical practice.

Much like the studies conducted by Atwood et al and Abu Dayyeh et al, a limitation to our study is the lack of dose measurements at greater distances from the stents. As Atwood and his colleagues astutely mentioned in their study, single sheets of film are sufficient and sensitive to measure dose patterns at the stent surface, but they do not yield information at tissue depth.

The International Commission on Radiation Units and Measurements (ICRU) recommends that radiation dose delivered to patients should be within $\pm 5 \%$ of the prescribed dose. We conclude that the dose perturbations caused by esophageal stents of varying designs and materials undergoing proton radiotherapy were not only negligible but also well within the ICRU's recommended range of $\pm 5 \%$.

\section{Conclusion}

Proton radiotherapy dose perturbations caused by stents of varying designs and material composition are negligible. Negligible dose perturbation is in keeping with the inherent advantage of proton therapy over traditional radiotherapy composed of photons - given their relatively large mass, protons have little side scatter. However, this interaction is yet to be confirmed in clinical practice. Additional clinical studies would clarify the benefit and safety profile of esophageal SEMS and SEPS among patients undergoing proton irradiation. Future studies should also examine the potential dose changes, if any, at greater distances from the stent/tissue interface.

\section{Competing interests: None}

\section{References}

1 American Cancer Society. Cancer Facts \& Figures 2012. Atlanta: American Cancer Society; 2012

2 American Cancer Society. Cancer Facts and Figures 2013. Atlanta: American Cancer Society; 2013

3 Javle M, Ailawadhi S, Yang GY et al. Palliation of malignant dysphagia in esophageal cancer: a literature based review. J Support Oncol 2006; 4: $365-373$

4 Minsky B. Choosing the correct modality for the treatment of dysphagia in the patient with esophageal cancer.J Support Oncol 2006; 4: 377

5 Urba S. Dysphagia: we can relieve this symptom. J Support Oncol 2006; 4: $378-379$

6 Coia LR, Soffen EM, Schultheiss TE et al. Swallowing function in patients with esophageal cancer treated with concurrent radiation and chemotherapy. Cancer 1993; 71: $281-286$

7 Li XA, Chibani O, Greenwald B et al. Radiotherapy dose perturbation of metallic esophageal stents. Int J Radiat Oncol Biol Phys 2002; 54: $1276-1285$

8 Chen YK, Schefter TE, Newman F. Esophageal cancer patients undergoing external beam radiation after placement of self-expandable metal stents: is there a risk of radiation dose enhancement? Gastrointest Endosc 2011; 73: 1109-1114

9 Atwood TF, Hsu A, Ogara MM et al. Radiotherapy dose perturbation of esophageal stents examined in an experimental model. Int J Radiat Oncol Biol Phys 2012; 82: 1659-1664

10 Abu Dayyeh BK, VanDamme JJ, Miller RC et al. Esophageal self-expandable stent material and mesh grid density are the major determining factors of external beam radiation dose perturbation: results from a phantom model. Endoscopy 2013; 45: $42-47$

11 Lee CT, Bilton SD, Famiglietti RM et al. Treatment planning with protons for pediatric retinoblastoma, medulloblastoma, and pelvic sarcoma: how do protons compare with other conformal techniques? Int J Radiat Oncol Biol Phys 2005; 63: $362-372$

12 Suit $H$, Urie M. Proton beams in radiation therapy. J Natl Cancer Inst 1992; 84: 155 - 164

13 Suit $H D$. Protons to replace photons in external beam radiation therapy? Clin Oncol (R Coll Radiol) 2003; 15: 29-S31

14 Weber DC, Rutz HP, Lomax AJ et al. First spinal axis segment irradiation with spot-scanning proton beam delivered in the treatment of a lumbar primitive neuroectodermal tumour. Case report and review of the literature. Clin Oncol (R Coll Radiol) 2004; 16: 326-331

15 Glimelius B, Isacsson $U$, Blomquist $E$ et al. Potential gains using high energy protons for therapy of malignant tumours. Acta Oncol 1999; 38: $137-145$

16 Seuntjens J, Olivares M, Evans $M$ et al. Absorbed dose to water reference dosimetry using solid phantoms in the context of absorbed-dose protocols. Med Phys 2005; 32: 2945-2953

17 Shin JH, Song HY, Kim JH et al. Comparison of temporary and permanent stent placement with concurrent radiation therapy in patients with esophageal carcinoma. J Vasc Interv Radiol 2005; 16: 67-74

18 Welsh J, Gomez D, Palmer MB et al. Intensity-modulated proton therapy further reduces normal tissue exposure during definitive therapy for locally advanced distal esophageal tumors: a dosimetric study. Int J Radiat Oncol Biol Phys 2011; 81: 1336-1342

19 Lin SH, Komaki R, Liao Z et al. Proton beam therapy and concurrent chemotherapy for esophageal cancer. Int J Radiat Oncol Biol Phys 2012; 83: e345-e351 